\title{
Diurnal variation of steroid hormones and their reference intervals using mass spectrometric analysis
}

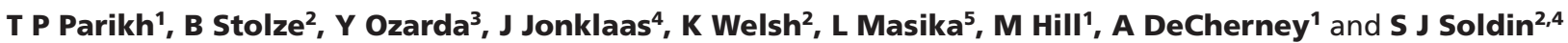 \\ ${ }^{1}$ Program in Reproductive and Adult Endocrinology, National Institute of Child Health and Human Development, National Institutes of Health (NIH), \\ Bethesda, Maryland, USA \\ 2Department of Laboratory Medicine, Clinical Center, NIH, Bethesda, Maryland, USA \\ ${ }^{3}$ Department of Medical Biochemistry, Faculty of Medicine, Uludag University, Bursa, Turkey \\ ${ }^{4}$ Division of Endocrinology and Metabolism, Department of Medicine, Georgetown University, Washington, District of Columbia, USA \\ ${ }^{5}$ Department of Laboratory Medicine and Pathology/National Health Laboratory Service Walter Sisulu University, Mthatha, South Africa \\ Correspondence should be addressed to S J Soldin: soldinsj@cc.nih.gov
}

\begin{abstract}
Objective: Accurate measurement of steroid hormones remains challenging. Mass spectrometry affords a reliable means for quantitating steroid profiles accurately. Our objective was to establish and define (1) the extent of diurnal fluctuations in steroid concentrations that potentially necessitate strict adherence to time of sample acquisition and (2) time-dependent steroid reference intervals.

Design: Nine steroid markers were examined in couplets in males and females.

Methods: Using isotope dilution high-performance liquid chromatography-tandem mass spectrometric (LC-MS/MS) analysis, we developed a multi-steroid profile requiring only a minimal volume of serum $(0.1 \mathrm{~mL})$. Couplet (AM and PM) measurements of steroid hormones for 120 healthy females (F) and 62 healthy males (M) were obtained. Patients were recruited from several participating centers.

Results: The following diurnal values were noted to be significantly different in both females and males: cortisone, cortisol, corticosterone, 11 deoxycortisol (11 DOC), androstenedione, 17a-hydroxyprogesterone (17 OHP) and dehydroepiandrosterone (DHEA). Testosterone was only found to have significant diurnal variance in males. Progesterone showed no significant difference in AM and PM values for either groups and thus may provide an internal control.

Conclusions: When diagnosing endocrine disorders, it is imperative to acknowledge the 24-h diurnal variation of the biochemical steroid markers. We highlight the importance of standardization of collection times and appropriate implementation of reference intervals. Precis: We identify diurnal fluctuations in steroid concentrations with time of day and emphasize the importance of adhering to firm time of sample acquisition.
\end{abstract}

\author{
Key Words \\ - mass spectrometry \\ - LC-MS/MS \\ - steroids \\ - diurnal variation
}

Endocrine Connections (2018) 7, 1354-1361

\section{Introduction}

Diurnal variations in the serum concentration of steroid hormones and their metabolism, as assessed by urinary excretion, have long been known for cortisol and testosterone $(1,2,3)$. Less information exists for the other up or downstream hormones within the steroid synthesis pathway $(4,5,6)$. A recent preliminary study comparing 8 AM and midnight samples on a small cohort of controls, suggests that significant diurnal variation in all but
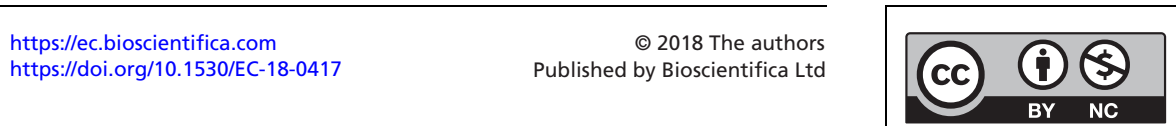

This work is licensed under a Creative Commons Attribution-NonCommercial 4.0 International License. 
progesterone probably does exist (7). Overall, we strongly believe the measurement of serum steroids is particularly important in endocrinology for an accurate evaluation of adrenal disorders and to make the distinction between pathologic processes and baseline adrenal, thyroid and gonadal functions of healthy individuals.

Appropriate reference intervals are essential to clinicians when interpreting laboratory results $(8,9)$. For years, groups such as the Clinical and Laboratory Standards Institute/International Federation of Clinical Chemistry (CLSI/IFCC), American Association of Clinical Chemistry, CALIPER and the Nordic Reference Interval Project have acknowledged this importance and developed guidelines by which researchers can ensure proper preanalytical and analytical testing methods $(10,11,12,13,14)$. The technical platforms on which biochemical samples are analyzed are immunoassay based and have been shown to report results which differ greatly between the differing platforms used (5).

The use of high-performance liquid chromatography (HPLC) in conjunction with tandem mass spectrometry (MS) is increasingly becoming the method of choice for clinical testing of hormone levels $(5,6,15,16,17)$. Given its high sensitivity, specificity and precision for the identification and detection of analytes, its use is fundamental to disease diagnosis, management and treatment outcomes. Most facilities use immunoassay techniques to measure steroid hormones and prior to this, radioimmunoassay methods were also often used $(18,19)$. Though HPLC-MS/MS is often criticized for its cost, poor access and need for trained personnel, many studies have found that it maintains better reproducibility, greater specificity and can be used to analyze multiple steroids simultaneously $(5,19,20,21)$. A recent systematic review of steroid hormones analyzed by MS highlights the impetus toward the use of advanced technologies for more accurate diagnosis (22). However, no consistency of time at which specimens were collected was found in these studies and can potentially be one of the many reasons for the wide range in reference intervals suggested by the various research groups (23).

Here, we demonstrate significant diurnal fluctuations of most of these aforementioned steroids in a suitable cohort of healthy controls to emphasize the need to maintain strict adherence to time of blood sample draw and requirement of time-dependent reference intervals. Simultaneous measurement of nine steroid hormones (cortisone, cortisol, corticosterone, 11 deoxycortisol, androstenedione, testosterone, 17a-hydroxyprogesterone, DHEA and progesterone) was performed using our previously published micro HPLC-MS/MS methods $(6,7)$. Diagnostic reliability is an important advantage gained by combining information for multiple steroids. Multiplexing several steroids (profiling) offers the ability to assay all of the important analytes from a single sample. Furthermore, we used samples from healthy community adults to establish time-dependent reference intervals for all nine analytes.

With the above considerations in mind, the present report provides the diurnal reference intervals for a panel of nine steroids measured in serum samples of healthy participants by an LC-MS/MS method primarily developed for diagnosis and subtyping of patients with disorders of adrenal function and steroidogenesis.

\section{Subjects and methods}

\section{Subjects and specimens}

Subjects included 182 healthy patients all providing appropriate informed consent. Healthy participants were determined by screening assessments and absence of evidence of any active or chronic disease following a detailed medical and surgical history. Healthy participants had stable body weight prior to testing, without medication or supplement use, and were enrolled following a history and physical examination by a medical provider. Serum samples were procured at the National Institutes of Health (NIH), Georgetown University, Walter Sisulu University and Uludag University. All samples in this study were approved by the institutional review board at NIH (protocol 93-CC-0094), National Institute of Diabetes and Digestive and Kidney Diseases/National Institute of Arthritis and Musculoskeletal and Skin Diseases Institutional Review Board (approved protocol NCT00428987) and the Georgetown University Institutional Review Board (Pro0000007-01).

Couplet measurements of steroid hormones were collected at $6 \mathrm{AM}-8 \mathrm{AM}$ and again at $6 \mathrm{PM}-8 \mathrm{PM}$ with 12-h sample intervals for analysis of individual steroid hormone profiles. Patients were informed that specimen collections and banking were for purposes of establishment of reference intervals for new diagnostic tests. Enrollment of subjects was facilitated by physician recruitment with a small financial reward to encourage involvement. Female subjects $(n=120)$ were aged $19-67$ years (mean 38.3, median 35$)$. Males subjects $(n=62)$ were aged $19-75$ years (mean 33.1, median 31). Participant demographic details as seen in Supplementary Tables 1 and 2 (see section on supplementary data given at the end of this article).

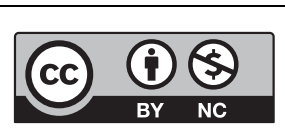

This work is licensed under a Creative Commons Attribution-NonCommercial 4.0 International License. 
Missing data for age were not incorporated in the reported mean and median values stated above.

All blood sampling was carried out by professional phlebotomists and collected in preservative/gel-free $10 \mathrm{~mL}$ plastic specimen collection tubes and kept chilled and centrifuged within $1 \mathrm{~h}$ of collection to separate serum, which was stored at $-80^{\circ} \mathrm{C}$ until assayed (BD Vacutainer Blood Collection Tubes, BD\#367820).

\section{LC-MS/MS}

Samples were analyzed using the Agilent 6490 triplequadrupole MS coupled with an atmospheric pressure photoionization source and Agilent 1200 Infinity series HPLC (Agilent Technologies). We used isotope dilution with deuterium-labeled internal standards for each analyte. Chemicals, reagents and methods of steroid hormone analysis in this study were used according to previously cited procedures (6). The tracebility and imprecision was evaluated by assaying Bio-Liquichek Plus Control replicates at two or three different concentration levels $(n=20)$ as described in our prior study (6).

The steroids in the panel included cortisone, cortisol, corticosterone, 11-deoxycortisol, androstenedione, testos terone, 17a-hydroxyprogesterone, DHEA and progesterone. Inter-laboratory quality and accuracy of sample analysis was assessed prior to the study with the Mayo Clinic (NMS Labs, Rochester, MN) and Children's National Medical Center (Washington, DC).

\section{Statistical analysis}

Statistical analyses were performed using Statistical Package for the Social Sciences, version 23 and box-and-whisker
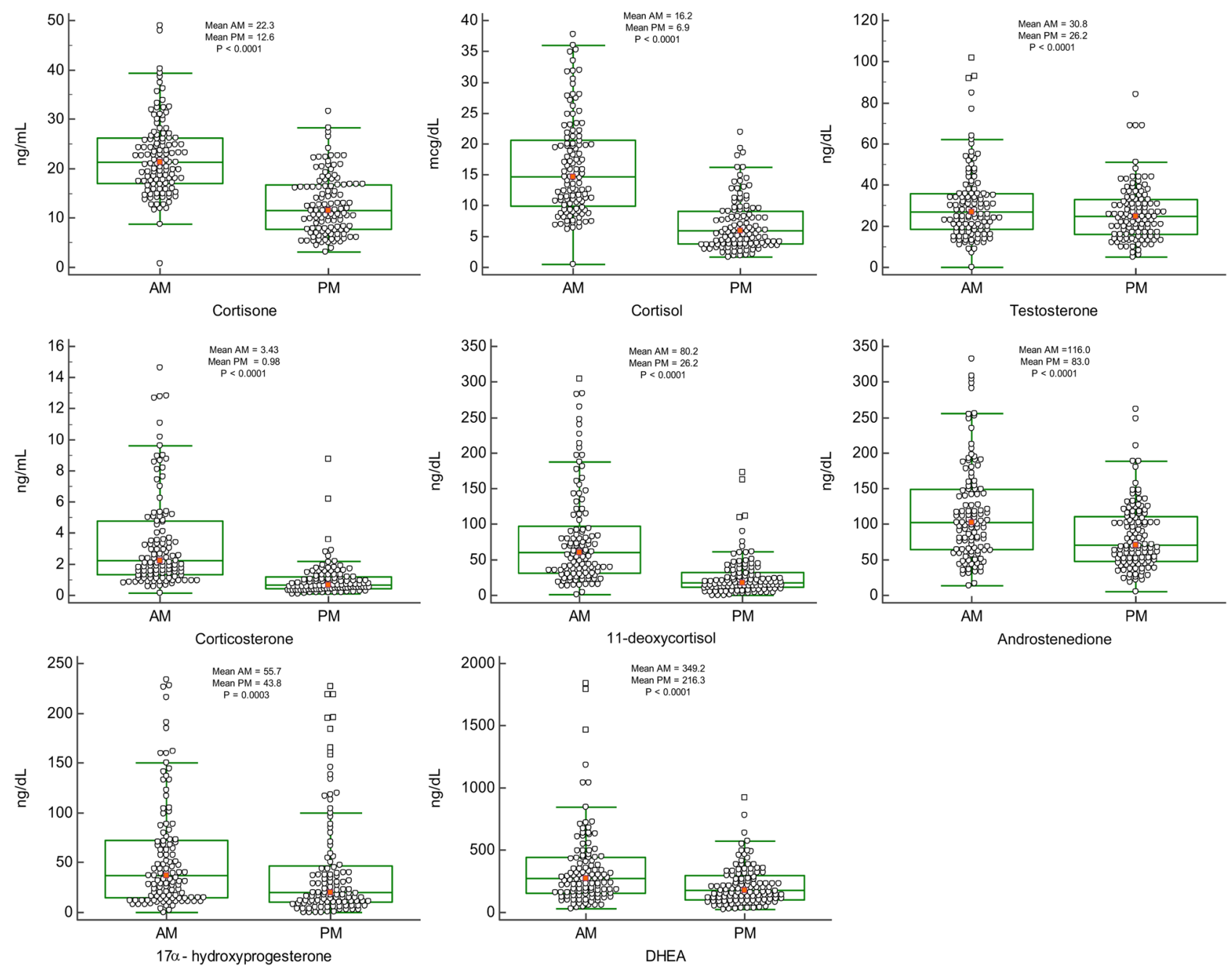

Figure 1

Jitter box-and-whisker plots: AM and PM steroid hormone values for female samples $(n=120)$. Mean is indicated by small square markers and median by bar in box, $P<0.0001$ ( $P<0.05$ is considered statistically significant).

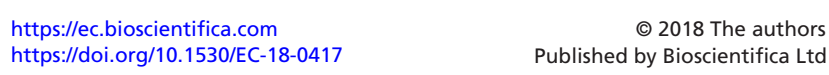


plots were generated using MedCalc, version 17.8.6. The normality of distribution of variables was tested by the Kolmogorov-Smirnov test and steroid hormone profiles were noted to be log normally distributed $(15,19)$. Reference intervals for two steroid hormones were obtained using the percentile approach. For this reason, data sets were analyzed with the paired Wilcoxon signed-rank test, pairing AM and PM values. Significant values were determined at $P<0.05$.

\section{Results}

The number of samples obtained from participants at the National Institutes of Health (NIH), Georgetown University, Walter Sisulu University and Uludag University were 76, 35, 47 and 24 respectively. Samples were collected between the period of 2016 and 2018.

Figures 1 and 2 are jitter box-and-whisker plots demonstrating the significant difference in paired samples of female $(n=120)$ and male $(n=62)$ AM/PM steroid levels respectively. Serum steroid levels between the females and males were also compared. However, no significant sex differences were seen for cortisone and corticosterone but significant sex differences were found for all the other steroids studied. Testosterone (elevated in males compared to females) and progesterone (elevated in females compared to males), differences were as expected. Our findings revealed significant diurnal fluctuations for cortisone, cortisol, corticosterone, 11-DOC, androstenedione, 17-OHP, DHEA and testosterone $(P<0.0001)$ showing significantly higher values in the
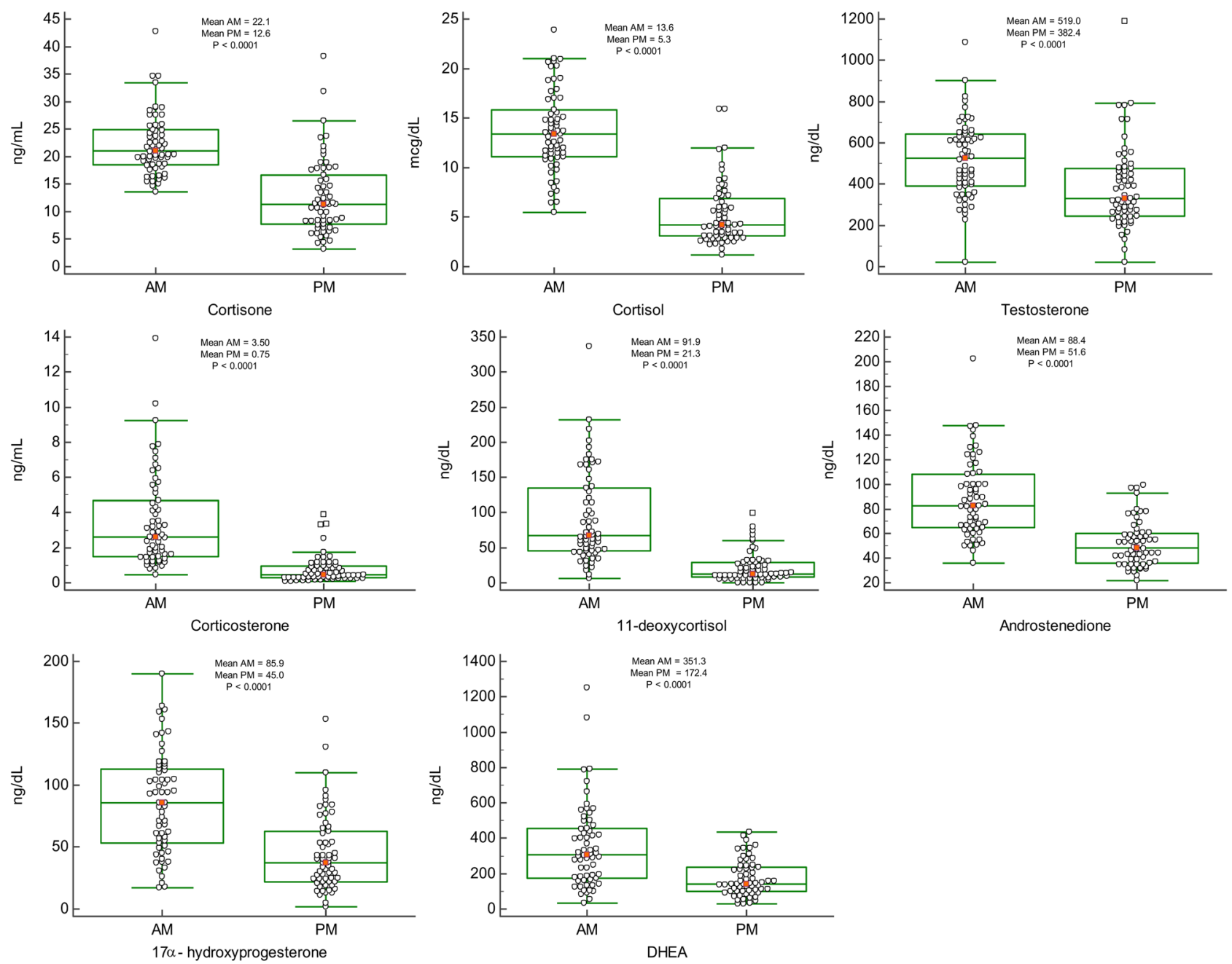

Figure 2

Jitter box-and-whisker plots: AM and PM steroid hormone values for male samples $(n=62)$. Mean is indicated by small square markers and median by bar in box, $P<0.0001$ ( $P<0.05$ is considered statistically significant).

https://ec.bioscientifica.com
https://doi.org/10.1530/EC-18-0417 2018 The authors


morning compared with evening collection times as seen in Figs 1, 2 and Table 1.

When specifically analyzing progesterone levels as seen in Fig. 3, women had a ten-fold higher mean progesterone level than their male counterparts. This is consistent with expected sex differences. No significant diurnal variation was seen in AM and PM measurements of progesterone in both males and female participants and thus serves as an internal control when analyzing the results of a steroid panel. For the women in this study, values acquired were not specific to phases of their menstrual cycles and therefore may be a potential reason why lack of variation was noted. Though studies have shown that progesterone levels do vary considerably depending on the follicular or luteal phase of the menstrual cycle, the focus of this study was to determine if diurnal variations were present (24). The dot and line graph in Fig. 3 represents the respective AM and PM progesterone level of each patient in a 24 -h period.

Table 2 shows the 2.5-97.5 percentile of the steroid hormone values grouped for females and males respectively. Additionally, two steroid hormones were similar in values between both female and male groups, such that they could be combined as seen in Supplementary Fig. 1. We thus provide recommended reference intervals for females and preliminary reference intervals for males to be used by laboratories routinely running this assay.

Table 3 demonstrates that regardless of the number of female participants, varying from a minimum of 60 to a maximum of 120 , the reference intervals were essentially found to be identical.

\section{Discussion}

Steroid hormones are routinely measured using automated direct immunoassays. This method of measurement is generally quick, inexpensive and does not require extensive training of personnel. Despite these advantages, new evidence has been emerging to advocate the improved specificity and accuracy of MS for analyzing steroid hormone profiles. We have developed a 3rd generation steroid profile assay utilizing an LC-APPI-MS/ MS based method for the analysis of nine steroids with a 7.0-min elution time and a two steroid 'backdoor' pathway method as previously described (6). This method requires small sample volumes and to date is the preferred way to measure steroid hormones accurately and precisely. Our prior study revealed similar diurnal patterns of fluctuation in DHEA alone with a smaller number of samples (male $n=9$ and female $n=10$ ) (6). Here, we see similar patterns of variation with time when analyzing other up and downstream steroid hormones.

Reference interval studies to date, fail to adequately control for this diurnal fluctuation and thus should be used with caution $(2,23,25)$. The most recent systematic review of published studies regarding reference intervals of steroid hormones mentions the possibility of variations in these hormones with time of day but does not suggest an approach to achieve time of day-adjusted values (22). Another study highlights the importance of age, sex, ethnicity and BMI for the establishment of pediatric steroid reference intervals, with no account of its diurnal

Table 1 Median interquartile ranges (IQR) for female $(n=120)$ and male $(n=62)$ participants.

\begin{tabular}{l} 
Steroid \\
\hline Cortisone AM \\
Cortisone PM \\
Cortisol AM \\
Cortisol PM \\
Corticosterone AM \\
Corticosterone PM \\
11-Deoxycortisol AM \\
11-Deoxycortisol PM \\
Androstenedione AM \\
Androstenedione PM \\
Testosterone AM \\
Testosterone PM \\
17-OHP AM \\
17-OHP PM \\
Progesterone AM \\
Progesterone PM \\
DHEA AM \\
DHEA PM
\end{tabular}

$\begin{array}{r}\hline \text { Median (IQR) female } \\ \hline 21.5(17.2-26.2) \\ 11.6(7.7-16.7) \\ 14.7(10.2-20.8) \\ 5.9(3.8-9.2) \\ 2.29(1.33-4.78) \\ 0.67(0.42-1.20) \\ 60.7(31.5-103.2) \\ 18.3(11.0-33.4) \\ 102.1(63.9-149.0) \\ 71.0(48.3-111.6) \\ 27.1(18.8-35.9) \\ 24.8(15.9-33.2) \\ 36.6(15.4-72.1) \\ 20.3(10.4-45.8) \\ 0.20(0.08-1.01) \\ 0.15(0.06-0.64) \\ 270.0(158.0-441.0) \\ 175.0(102.0-295.0) \\ \hline\end{array}$

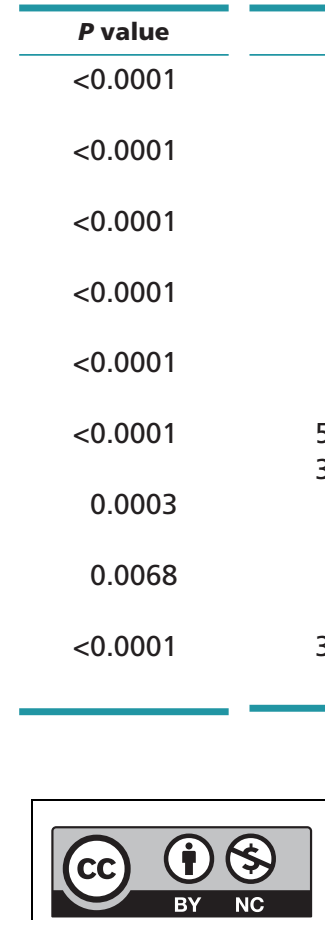

Median (IQR) male
$21.1(18.5-24.9)^{\mathrm{a}}$
$11.3(7.8-16.5)^{\mathrm{a}}$
$13.4(11.1-15.7)$
$4.3(3.1-6.8)$
$2.63(1.53-4.63)^{\mathrm{a}}$
$0.47(0.28-0.96)^{\mathrm{a}}$
$66.9(46.0-133.9)$
$12.2(7.8-28.8)$
$83.0(65.3-106.3)$
$48.5(36.6-60.2)$
$524.4(393.2-637.7)$
$329.3(249.0-473.8)$
$86.2(53.5-113.1)$
$37.5(22.5-62.8)$
$0.09(0.04-0.16)$
$0.04(0.00-0.12)$
$308.0(175.0-456.0)$
$141.0(99.0-233.8)$

$\boldsymbol{P}$ value

$<0.0001$

$<0.0001$

$<0.0001$

$<0.0001$

$<0.0001$

$<0.0001$

$<0.0001$

0.2936

$<0.0001$

aCombined male and female data $(n=182)$.

$$
\begin{array}{lr}
\text { https://ec.bioscientifica.com } & \text { () } 2018 \text { The authors } \\
\text { https://doi.org/10.1530/EC-18-0417 } & \text { Published by Bioscientifica Ltd }
\end{array}
$$



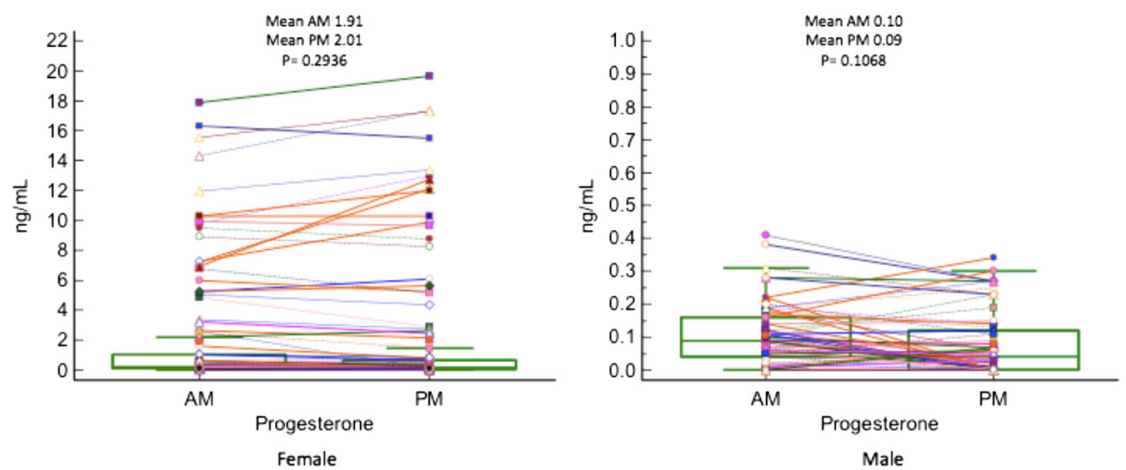

Figure 3

Dot and Line plot: AM and PM progesterone hormone values for female $(n=120)$ and male $(n=62)$ samples. variation (26). Here, we confirm our prior findings with a far larger cohort of patients to emphasize the importance of this time-dependent bimodal distribution and the need for reference intervals to acknowledge and reflect these findings. In terms of amplitude and timing variability, our results for all steroids tested are consistent with those of previous investigations $(2,3,4,15,16,27)$. A drawback of most other steroid reference interval studies $(15,23,26)$ is their lack of use of atmospheric pressure photoionization (APPI) as the ionization source and this results in increased levels of ionization inhibition thus making their data less credible (28). The use of both AM and PM steroid measurements from a single individual sufficiently enables the use of paired analysis with internal standardization.

Although the importance of reference intervals has been highlighted here, it should be recognized that the future of optimization of laboratory methodologies today lies in its importance to advancing individualized care. Steroid profile monitoring is more specifically advantageous as many endocrine disorders affect multiple steroids and typically abnormalities are not isolated (29). Historically, personalized or precision medicine has been restricted to select or optimize a patient's preventive and therapeutic care depending on the patients genetic evaluation or oncologic diagnosis $(30,31)$. Molecular profiling in healthy patients or patients with strong family histories of endocrinopathies may allow for earlier detection of abnormalities. This potentially enables physicians to offer a more comprehensive form of personalized medicine than is presently afforded. The detection of subtle changes in baseline metabolic profiles may be achieved with such technologies as the LC-APPI-MS/MS and may be used to customize medical care to that individual's future needs.

We openly acknowledge that the limitations of this study include a lower than anticipated recruitment of healthy male participants. Female participant recruitment does meet the recommended 120 subjects per the IFCC (32). Despite these arguments, an attempt to demonstrate the validity of our data even with our low recruitment of healthy male patients was made in Table 3. As shown, whether the number of participants analyzed were $n=60$, 90 or 120 , references ranges remained minimally changed. Additionally, the significant difference in variation of steroid hormones from AM to PM continued to be present. We further acknowledge that progesterone values should have been collected with respect to follicular or secretory phase of the participant's menstrual cycle; however, we note that this was not the primary study outcome and caution that progesterone variation may be present

Table 2 Recommended female reference ranges.

\begin{tabular}{|c|c|c|c|c|c|c|c|c|}
\hline \multirow[b]{3}{*}{ Percentile approach } & \multicolumn{4}{|c|}{ Female $(n=120)$} & \multicolumn{4}{|c|}{ Male $(n=62)^{\mathbf{a}}$} \\
\hline & \multicolumn{2}{|c|}{$\mathrm{AM}(\%)$} & \multicolumn{2}{|c|}{ PM (\%) } & \multicolumn{2}{|c|}{ AM (\%) } & \multicolumn{2}{|c|}{ PM (\%) } \\
\hline & 2.5 & 97.5 & 2.5 & 97.5 & 2.5 & 97.5 & 2.5 & 97.5 \\
\hline Cortisone (ng/mL) & 12.0 & 39.4 & 5.0 & 26.5 & $14.6^{b}$ & $34.6^{b}$ & $4.3^{b}$ & $31.8^{\mathrm{b}}$ \\
\hline Cortisol ( $\mu \mathrm{g} / \mathrm{dL})$ & 6.5 & 34.9 & 2.0 & 18.1 & 6.4 & 21.0 & 1.8 & 15.9 \\
\hline Corticosterone (ng/mL) & 0.61 & 12.70 & 0.17 & 2.90 & $0.77^{b}$ & $10.20^{b}$ & $0.10^{b}$ & $3.34^{\mathrm{b}}$ \\
\hline $11-D O C(n g / d L)$ & 13.3 & 264.9 & 0.7 & 110.0 & 11.0 & 232.1 & 0.0 & 79.8 \\
\hline Androstenedione (ng/dL) & 30.8 & 298.4 & 22.4 & 202.0 & 46.0 & 148.2 & 26.0 & 97.0 \\
\hline Testosterone (ng/dL) & 9.0 & 84.8 & 8.2 & 69.0 & 227.0 & 903.4 & 82.0 & 790.0 \\
\hline 17-OHP (ng/dL) & 6.8 & 216.4 & 0.0 & 196.0 & 18.0 & 164.0 & 5.0 & 131.0 \\
\hline Progesterone (ng/mL) & 0.0 & 14.31 & 0.0 & 15.50 & 0.0 & 0.38 & 0.0 & 0.30 \\
\hline DHEA (ng/dL) & 59.4 & 1185.0 & 35.0 & 574.0 & 51.5 & 1080.0 & 30.8 & 414.0 \\
\hline \multicolumn{9}{|c|}{ aproposed male reference ranges; ${ }^{\text {b}}$ combined male and female data $(n=182)$. } \\
\hline $\begin{array}{l}\text { https://ec.bioscientifica.com } \\
\text { https://doi.org/10.1530/EC-18-0417 }\end{array}$ & \multicolumn{3}{|c|}{$\begin{array}{l}\text { () } 2018 \text { The authors } \\
\text { by Bioscientifica Ltd }\end{array}$} & & \multicolumn{4}{|c|}{$\begin{array}{l}\text { This work is licensed under a Creative Commons } \\
\text { Attribution-NonCommercial } 4.0 \text { International } \\
\text { License. }\end{array}$} \\
\hline
\end{tabular}


Table 3 Female reference ranges of $n=60, n=98, n=120$.

\begin{tabular}{l} 
Female \\
\hline Cortisone $(\mathrm{ng} / \mathrm{mL})$ \\
Cortisol $(\mu \mathrm{g} / \mathrm{dL})$ \\
Corticosterone $(\mathrm{ng} / \mathrm{mL})$ \\
$11-D O C(\mathrm{ng} / \mathrm{dL})$ \\
Androstenedione $(\mathrm{ng} / \mathrm{dL})$ \\
Testosterone $(\mathrm{ng} / \mathrm{dL})$ \\
$17-O H P(\mathrm{ng} / \mathrm{dL})$ \\
Progesterone $(\mathrm{ng} / \mathrm{mL})$ \\
DHEA $(\mathrm{ng} / \mathrm{dL})$
\end{tabular}

\begin{tabular}{|c|c|c|}
\hline \multicolumn{3}{|c|}{ AM } \\
\hline & $2.5 \%$ & \\
\hline$n=60$ & $n=98$ & $n=120$ \\
\hline 12.7 & 11.7 & 12.0 \\
\hline 7.0 & 6.4 & 6.5 \\
\hline 0.74 & 0.58 & 0.61 \\
\hline 15.9 & 13.0 & 13.3 \\
\hline 33.0 & 31.0 & 30.8 \\
\hline 7.0 & 9.0 & 9.0 \\
\hline 9.0 & 4.0 & 6.8 \\
\hline 0.00 & 0.00 & 0.00 \\
\hline 50.2 & 50 & 59.4 \\
\hline \multicolumn{3}{|c|}{ PM } \\
\hline \multicolumn{3}{|c|}{$2.5 \%$} \\
\hline$n=60$ & $n=98$ & $n=120$ \\
\hline 5.0 & 5.0 & 5.0 \\
\hline 1.9 & 2.0 & 2.0 \\
\hline 0.11 & 0.20 & 0.17 \\
\hline 2.5 & 0.0 & 0.7 \\
\hline 24.3 & 22.0 & 22.4 \\
\hline 6.0 & 8.0 & 8.2 \\
\hline 2.7 & 0.0 & 0.0 \\
\hline 0.00 & 0.00 & 0.00 \\
\hline 35.0 & 31.0 & 35.0 \\
\hline
\end{tabular}

\begin{tabular}{|c|c|c|}
\hline \multicolumn{3}{|c|}{ AM } \\
\hline & $97.5 \%$ & \\
\hline$n=60$ & $n=98$ & $n=120$ \\
\hline 40.2 & 40.2 & 39.4 \\
\hline 31.8 & 35.3 & 34.9 \\
\hline 12.84 & 12.70 & 12.70 \\
\hline 283.8 & 283.0 & 264.9 \\
\hline 298.4 & 304.0 & 298.4 \\
\hline 76.5 & 85.0 & 84.8 \\
\hline 226.4 & 226.0 & 216.4 \\
\hline 16.30 & 11.94 & 14.31 \\
\hline 1185.0 & 1470.0 & 1185.0 \\
\hline \multicolumn{3}{|c|}{ PM } \\
\hline \multicolumn{3}{|c|}{$97.5 \%$} \\
\hline$n=60$ & $n=98$ & $n=120$ \\
\hline 27.3 & 27.3 & 26.5 \\
\hline 18.1 & 18.6 & 18.1 \\
\hline 2.80 & 2.90 & 2.90 \\
\hline 90.1 & 112.0 & 110.0 \\
\hline 210.5 & 180.0 & 202.0 \\
\hline 69.0 & 69.0 & 69.0 \\
\hline 218.9 & 219.0 & 196.0 \\
\hline 15.50 & 13.40 & 15.50 \\
\hline 574.0 & 550.0 & 574.0 \\
\hline
\end{tabular}

\section{Cortisone $(\mathrm{ng} / \mathrm{mL})$}

Cortisol $(\mu \mathrm{g} / \mathrm{dL})$

Corticosterone (ng/mL)

$11-\mathrm{DOC}(\mathrm{ng} / \mathrm{dL})$

Androstenedione $(\mathrm{ng} / \mathrm{dL})$

Testosterone (ng/dL)

17-OHP (ng/dL)

Progesterone $(\mathrm{ng} / \mathrm{mL})$

DHEA (ng/dL)

Male reference range $(n=62)$ should be acceptable.

from AM to PM if further subcategorization of patients individual phase of cycle were performed.

In summary, this study emphasizes the time- and genderspecific variations for LC-MS/MS-derived measurements of plasma concentrations for nine steroids in a panel designed primarily for investigation of healthy patients. Our study emphasizes the need for time specific reference intervals given the significant diurnal variations seen with this study and these reference intervals were implemented at the Clinical Center, NIH in December 2017. We urge other institutions to strongly consider incorporating reference intervals to reflect this diurnal variation and sex-dependent differences.

\section{Supplementary data}

This is linked to the online version of the paper at https://doi.org/10.1530/ EC-18-0417.

\section{Declaration of interest}

Author S S is an inventor on U.S. Patent US7473560. The other authors have nothing to declare.

\section{Funding}

J J was funded through Grant \# UL1TR001409 from the National Center for Advancement of Translational Science and S S was funded through NIH intramural protocol NCT00428987. Support for the portion of the study performed at Georgetown University was provided by Grant \# UL1TR001409 from the National Center for Advancement of Translational
Science, NIH, through the Clinical and Translational Science Awards Program (CTSA), a trademark of DHHS, part of the Roadmap Initiative, 'Re Engineering the Clinical Research Enterprise'. S S was supported with funds from the NIH Intramural Research Grant.

\section{Acknowledgements}

This research has been supported (in part) by the Intramural program of the $\mathrm{NIH}$, Georgetown University, Walter Sisulu University and Uludag University.

\section{References}

1 Bartels M, Van den Berg M, Sluyter F, Boomsma D \& de Geus EJ. Heritability of cortisol levels: review and simultaneous analysis of twin studies. Psychoneuroendocrinology 200328 121-137. (https://doi. org/10.1016/S0306-4530(02)00003-3)

2 Brambilla DJ, Matsumoto AM, Araujo AB \& McKinlay JB. The effect of diurnal variation on clinical measurement of serum testosterone and other sex hormone levels in men. Journal of Clinical Endocrinology and Metabolism 200994 907-913. (https://doi.org/10.1210/jc.2008-1902)

3 Migeon CJ, Tyler FH, Mahoney JP, Florentin AA, Castle H, Bliss EL $\&$ Samuels LT. The diurnal variation of plasma levels and urinary excretion of 17-hydroxycorticosteroids in normal subjects, night workers and blind subjects. Journal of Clinical Endocrinology and Metabolism 195616 622-633. (https://doi.org/10.1210/jcem-16-5-622)

4 Doe RP, Vennes JA, Flink EB, Engst R, Johnson P \& Lewis M. Diurnal variation of 17-hydroxycorticosteroids, sodium, potassium, magnesium and creatinine in normal subjects and in cases of treated adrenal insufficiency and Cushing's syndrome. Journal of Clinical Endocrinology and Metabolism 196020 253-265. (https://doi. org/10.1210/jcem-20-2-253)

5 Soldin SJ \& Soldin OP. Steroid hormone analysis by tandem mass spectrometry. Clinical Chemistry 200955 1061-1066. (https://doi. org/10.1373/clinchem.2007.100008)

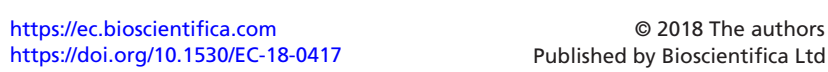


6 Stolze BR, Gounden V, Gu J, Elliott EA, Masika LS, Abel BS, Merke DP, Skarulis MC \& Soldin SJ. An improved micro-method for the measurement of steroid profiles by APPI-LC-MS/MS and its use in assessing diurnal effects on steroid concentrations and optimizing the diagnosis and treatment of adrenal insufficiency and CAH. Journal of Steroid Biochemistry and Molecular Biology 2016162 110-116. (https://doi.org/10.1016/j.jsbmb.2015.12.024)

7 Stolze BR, Gounden V, Gu J, Abel BS, Merke DP, Skarulis MC \& Soldin SJ. Use of micro-HPLC-MS/MS method to assess diurnal effects on steroid hormones. Clinical Chemistry 201561 556-558. (https:// doi.org/10.1373/clinchem.2014.232546)

8 Aytekin M \& Emerk K. Accurate reference intervals are required for accurate diagnosis and monitoring of patients. Electronic Journal of IFCC 200819137.

9 Kushnir MM, Rockwood AL, Roberts WL, Yue B, Bergquist J \& Meikle AW. Liquid chromatography tandem mass spectrometry for analysis of steroids in clinical laboratories. Clinical Biochemistry 2011 44 77-88. (https://doi.org/10.1016/j.clinbiochem.2010.07.008)

10 Aw T-C, Phua S-K \& Tan S-P. Measurement of cardiac troponin I in serum with a new high-sensitivity assay in a large multi-ethnic Asian cohort and the impact of gender. Clinica Chimica Acta $2013 \mathbf{4 2 2}$ 26-28. (https://doi.org/10.1016/j.cca.2013.03.034)

11 Estey MP, Cohen AH, Colantonio DA, Chan MK, Marvasti TB, Randell E, Delvin E, Cousineau J, Grey V \& Greenway D. CLSI-based transference of the CALIPER database of pediatric reference intervals from Abbott to Beckman, Ortho, Roche and Siemens Clinical Chemistry Assays: direct validation using reference samples from the CALIPER cohort. Clinical Biochemistry 201346 1197-1219. (https:// doi.org/10.1016/j.clinbiochem.2013.04.001)

12 Fraser CG. Pediatric reference ranges. Steven J. Soldin, Barlo Brugnara \& Edward C. Wong, editors. Washington DC: AACC Press, 2003, 248 pp., \$67.00 (AACC members \$55.00). ISBN 1-890883-88-3. Clinical Chemistry 200450 262-263. (https://doi.org/10.1373/ clinchem.2003.025882)

13 Horowitz GL, Altaie S \& Boyd JC. Defining, Establishing, and Verifying Reference Intervals in the Clinical Laboratory; Approved Guideline. Wayne, PA, USA: Clinical and Laboratory Standards Insititute, 2010.

14 Katayev A, Balciza C \& Seccombe DW. Establishing reference intervals for clinical laboratory test results: is there a better way? American Journal of Clinical Pathology 2010133 180-186. (https://doi. org/10.1309/AJCPN5BMTSF1CDYP)

15 Eisenhofer G, Peitzsch M, Kaden D, Langton K, Pamporaki C, Masjkur J, Tsatsaronis G, Mangelis A, Williams TA \& Reincke M. Reference intervals for plasma concentrations of adrenal steroids measured by LC-MS/MS: impact of gender, age, oral contraceptives, body mass index and blood pressure status. Clinica Chimica Acta 2017470 115-124. (https://doi.org/10.1016/j.cca.2017.05.002)

16 Guo T, Taylor RL, Singh RJ \& Soldin SJ. Simultaneous determination of 12 steroids by isotope dilution liquid chromatography-photospray ionization tandem mass spectrometry. Clinica Chimica Acta 2006372 76-82. (https://doi.org/10.1016/j.cca.2006.03.034)

17 Stanczyk FZ \& Clarke NJ. Advantages and challenges of mass spectrometry assays for steroid hormones. Journal of Steroid Biochemistry and Molecular Biology 2010121 491-495. (https://doi. org/10.1016/j.jsbmb.2010.05.001)

18 Chang Y-C, Li C-M, Li L-A, Jong S-B, Liao P-C \& Chang LW. Quantitative measurement of male steroid hormones using automated on-line solid phase extraction-liquid chromatographytandem mass spectrometry and comparison with radioimmunoassay. Analyst 2003128 363-368. (https://doi.org/10.1039/b210111b)
19 Dorgan JF, Fears TR, McMahon RP, Aronson Friedman L, Patterson BH \& Greenhut SF. Measurement of steroid sex hormones in serum: a comparison of radioimmunoassay and mass spectrometry. Steroids 200267 151-158. (https://doi.org/10.1016/ S0039-128X(01)00147-7)

20 Minutti CZ, Lacey JM, Magera MJ, Hahn SH, McCann M, Schulze A, Cheillan D, Dorche C, Chace DH \& Lymp JF. Steroid profiling by tandem mass spectrometry improves the positive predictive value of newborn screening for congenital adrenal hyperplasia. Journal of Clinical Endocrinology and Metabolism 200489 3687-3693. (https:// doi.org/10.1210/jc.2003-032235)

21 Shackleton C. Clinical steroid mass spectrometry: a 45-year history culminating in HPLC-MS/MS becoming an essential tool for patient diagnosis. Journal of Steroid Biochemistry and Molecular Biology 2010 121 481-490. (https://doi.org/10.1016/j.jsbmb.2010.02.017)

22 Greaves RF. Systematic review of serum steroid reference intervals developed using mass spectrometry. Clinical Biochemistry 201750 1260-1274. (https://doi.org/10.1016/j.clinbiochem.2017.07.002)

23 Kyriakopoulou L, Yazdanpanah M, Colantonio DA, Chan MK, Daly CH \& Adeli K. A sensitive and rapid mass spectrometric method for the simultaneous measurement of eight steroid hormones and CALIPER pediatric reference intervals. Clinical Biochemistry 201346 642-651. (https://doi.org/10.1016/j.clinbiochem.2013.01.002)

24 Check JH, Nowroozi K, Check ML \& Vetter B. Correlation of serum progestagen-associated endometrial protein levels with endometrial biopsies serum steroid levels and therapy for luteal phase defects. Human Reproduction 19916 511-514. (https://doi.org/10.1093/ oxfordjournals.humrep.a137369)

25 Gounden V \& Soldin SJ. Clinical use of reference intervals derived from some CALIPER studies questioned. Clinical Chemistry 201460 416-417. (https://doi.org/10.1373/clinchem.2013.214684)

26 Bailey D, Colantonio D, Kyriakopoulou L, Cohen AH, Chan MK, Armbruster D \& Adeli K. Marked biological variance in endocrine and biochemical markers in childhood: establishment of pediatric reference intervals using healthy community children from the CALIPER cohort. Clinical Chemistry 201359 1393-1405. (https://doi. org/10.1373/clinchem.2013.204222)

27 Alary J. Comparative study: LC/MS/MS analysis of four steroid compounds using a new photoionization source and a conventional APCI source. In Proceedings of the 49th ASMS Conference on Mass Spectrometry and Allied Topics, pp 27-31. Chicago, IL, USA: ASMA, 2001.

28 Sun Q, Gu J, Stolze BR \& Soldin SJJCc. Atmospheric pressure chemical ionization is a suboptimal ionization source for steroids. Clinical Chemistry 201864 974-976. (https://doi.org/10.1373/ clinchem.2018.287029)

29 Holst JP, Soldin SJ, Tractenberg RE, Guo T, Kundra P, Verbalis JG \& Jonklaas J. Use of steroid profiles in determining the cause of adrenal insufficiency. Steroids $2007 \mathbf{7 2}$ 71-84. (https://doi.org/10.1016/j. steroids.2006.11.001)

30 Duarte TT \& Spencer CT. Personalized proteomics: the future of precision medicine. Proteomes 20164 29. (https://doi.org/10.3390/ proteomes4040029)

31 Weitzel JN, Blazer KR, MacDonald DJ, Culver JO \& Offit K. Genetics, genomics and cancer risk assessment: state of the art and future directions in the era of personalized medicine. CA: A Cancer Journal for Clinicians 201161 327-359. (https://doi. org/10.3322/caac.20128)

32 Horowitz GL. Reference intervals: practical aspects. Electronic Journal of IFCC 200819 95-105.
Received in final form 1 October 2018

Accepted 24 October 2018

Accepted Preprint published online 26 October 2018 